\title{
Formation of Acetaldehyde on CO-rich Ices
}

\author{
Thanja Lamberts, ${ }^{*, \dagger, \ddagger}$ Max N. Markmeyer, ${ }^{\dagger}$ Florian J. Kolb, ${ }^{\dagger}$ and Johannes \\ Kästner*,† \\ $\dagger$ Institute for Theoretical Chemistry, University of Stuttgart, Pfaffenwaldring 55, 70569 \\ Stuttgart, Germany \\ $\ddagger$ Leiden Institute of Chemistry, Gorlaeus Laboratories, Leiden University, P.O. Box 9502, \\ 2300 RA Leiden, The Netherlands \\ E-mail: a.I.m.lamberts@lic.leidenuniv.nl; kaestner@theochem.uni-stuttgart.de
}

\begin{abstract}
The radicals $\mathrm{HCO}$ and $\mathrm{CH}_{3}$ on carbon monoxide ice surfaces were simulated using density functional theory. Their binding energy on amorphous CO ice shows broad distributions, with approximative average values of $500 \mathrm{~K}$ for $\mathrm{HCO}$ and $200 \mathrm{~K}$ for $\mathrm{CH}_{3}$. If they are located on the surface close to each other ( 3 to $4 \AA$ ), molecular dynamics calculations based on density functional theory show that they can form acetaldehyde $\left(\mathrm{CH}_{3} \mathrm{CHO}\right)$ or $\mathrm{CH}_{4}+\mathrm{CO}$ in barrier-less reactions, depending on the initial orientation of the molecules with respect to each other. In some orientations, no spontaneous reactions were found, the products remained bound to the surface. Sufficient configurational sampling, inclusion of the vibrational zero point energy, and a thorough benchmark of the applied electronic structure method are important to predict reliable binding energies for such weakly interacting systems. From these results it is clear that complex organic molecules, like acetaldehyde, can be formed by recombination reactions of radicals on $\mathrm{CO}$ surfaces.
\end{abstract}


Keywords: interstellar medium, complex organic molecules, CO ice, radical-radical recombination, binding energy

\section{Introduction}

Acetaldehyde is a major precursor for several complex organic molecules (COMs) in astrochemical surface reactions. It has first been detected in the interstellar medium in the 70's of the previous century ${ }^{1 / 3}$ and thereafter it has also been detected in other cold clouds, TMC-1 and L134N, ${ }^{4}$ translucent clouds ${ }^{5 / 7}$ and pre-stellar cores. ${ }^{[89}$ In CO rich ices on the surfaces of dust grains, the direct hydrogenation of $\mathrm{CO}$ leads to the closed-shell molecules formaldehyde and methanol. En route to these, radicals such as HCO are formed. Larger molecules that exhibit an additional $\mathrm{C}-\mathrm{C}$ bond are believed to be formed, at least partially, by radical-radical recombination reactions at low temperatures.10111 In other words, when two radicals find each other it is often assumed that a barrier-less reaction can take place leading to the formation of larger closed-shell molecules. Radicals can encounter each other either via creation in the ice next to each other, ${ }^{[12}$ via thermally induced diffusion ${ }^{[10}$ or via hot diffusion following reaction. $\frac{13}{13}$ The radical-radical recombination of $\mathrm{HCO}$ and $\mathrm{CH}_{3}$ can lead to acetaldehyde, but can also lead to $\mathrm{CH}_{4}+\mathrm{CO}$, i.e., no formation of a new $\mathrm{C}-\mathrm{C}$ bond. For the similar reaction $\mathrm{HCO}+\mathrm{NH}_{2}$ on a water ice surface it was recently shown that the outcome depends on the orientation of the radicals with respect to each other.14

The solid-state formation of acetaldehyde has experimentally been confirmed following UV-irradiation of methanol ices,,$\frac{1516}{16}$ albeit in low quantities. One possible reaction mechanism is through the $\mathrm{C}-\mathrm{C}$ bond formation via a reaction between $\mathrm{CH}_{3}$ and $\mathrm{HCO}$ radicals. This reaction, however, can also take place without additional energetic input of UV-irradiation, as long as both radicals are available. This may indeed be the case during the later stages of the cold dense clouds, when more $\mathrm{CO}$ is available and hydrogenation of carbon monoxide results in $\mathrm{HCO}$ formation. In either case, methanol photodissociation or non-energetic pro- 
cessing, such a reaction is expected to take place in an ice matrix that is no longer water dominated. 17

In this work we show that in a CO-rich environment indeed the formation of acetaldehyde from $\mathrm{HCO}$ and $\mathrm{CH}_{3}$ is possible. However, when these radicals meet, another product channel leading to $\mathrm{CH}_{4}+\mathrm{CO}$ is also open. Furthermore, we show that the outcome depends on the relative orientation of the two radicals with respect to each other. This is the first ab initio computational study of a radical-radical reaction on a CO ice cluster. As such this is also a comment on the importance of taking into account other ice constituents than only $\mathrm{H}_{2} \mathrm{O}$.

\section{Methodology}

The methods to simulate the radical-radical reaction are comprised of three steps: 1) the construction of a $\mathrm{CO}$ cluster, 2) the determination of $\mathrm{HCO}$ and $\mathrm{CH}_{3}$ binding sites and corresponding binding energies, and 3) the direct simulation of the radical-radical surface reactions $\mathrm{HCO}+\mathrm{CH}_{3}$. For all calculations density functional theory (DFT) has been used within the program ChemShell. $\stackrel{1819}{ }$ Both molecular dynamics (MD) runs and geometry optimizations were carried out within this framework. Geometry optimizations were performed with the DL-find library. ${ }^{20}$ The PBEh-3c functional in combination with the def2-mSVP basis set ${ }^{21}$ in turbomole ${ }^{22}$ are used for all MD runs as well as for the geometry optimizations. Refinements of the binding sites and binding energies are also calculated with the M06-2X functional ${ }^{23}$ and def2-TZVPD basis set ${ }^{24 \mid 25}$ including a Grimme's D3 correction. ${ }^{26}$ Finally we provide a small benchmark study at the CCSD(T)-F12/VTZ-F12 level of theory ${ }^{27}$ [1] using molpro ${ }^{32}$ to get a feeling for the error bar on the binding energies.

\section{Construction of the $\mathrm{CO}$ cluster}

A cluster consisting of $17 \mathrm{CO}$ molecules was prepared using Avogadro ${ }^{33}$ and subsequently relaxed via a short ab initio $\mathrm{MD}$ run at the $\mathrm{PBEh}-3 \mathrm{c} / \mathrm{mSVP}$ level of theory, followed by 


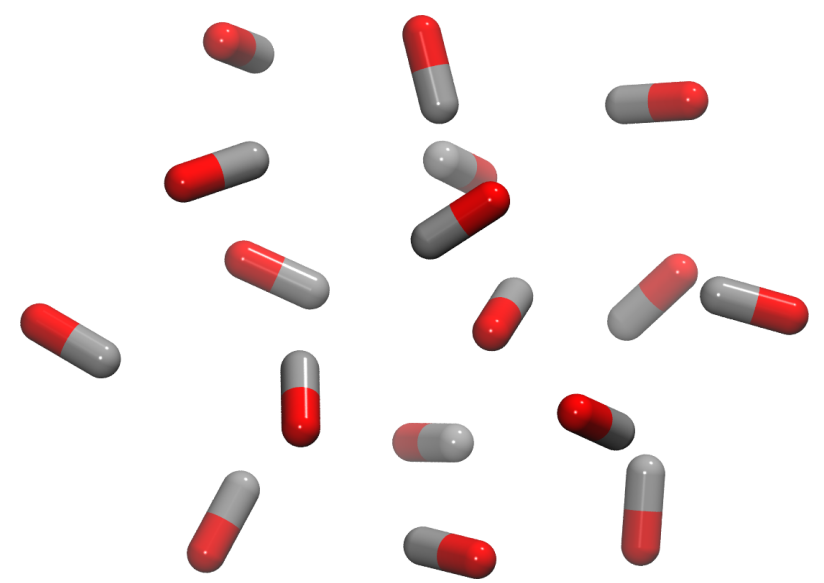

Figure 1: The bare CO cluster used in this study.

geometry optimization. In this way, an amorphous ellipsoidal structure of approximately $12 \times 8 \times 8 \AA^{3}$ is obtained, allowing for a variety of binding sites that is used throughout the calculations. The geometry of the resulting cluster is shown in Figure 1 .

\section{$\mathrm{HCO}$ and $\mathrm{CH}_{3}$ binding sites}

For each radical, $\mathrm{HCO}$ and $\mathrm{CH}_{3}$, the binding site distribution was probed by generating 60 different positions of the radical arranged symmetrically at the vertices of a truncated icosahedron at a distance of approximately 3-4 $\AA$ above the surface around the entire cluster. Geometry optimization at the PBEh-3c/mSVP level of theory was then performed resulting in 60 binding sites. Binding energies were determined via the difference in energy between the adsorbed species on the cluster and the separated radical and cluster. In other words, the relaxation of the surface is included in the binding energy. We used the convention that positive binding energies refer to exothermic binding. Subsequently, binding energies at the M06-2X/TZVPD+D3 level of theory were calculated of the previously optimized structures.

Various different binding modes for both radicals were found. Three representative geometries were selected for $\mathrm{HCO}$ and two for $\mathrm{CH}_{3}$ for which the harmonic zero-point vibrational energy (ZPE) correction to the binding energy was calculated with PBEh-3c/mSVP. The coordinates of these representative structures are provided in the Supporting Informa- 
tion. These specific five geometries were then further refined through a reoptimization with M06-2X/TZVPD+D3. During this reoptimization, to reduce the computational cost, all but the four $\mathrm{CO}$ molecules closest to the adsorbant were kept frozen.

\section{Surface reaction of $\mathrm{HCO}+\mathrm{CH}_{3}$}

The surface reaction of $\mathrm{HCO}$ with $\mathrm{CH}_{3}$ was studied through MD simulations in the microcanonical ensemble (NVE). Low random initial velocities, corresponding to a temperature of $10 \mathrm{~K}$, were assigned to restrict the study to barrier-less processes. A small time step of 0.2 fs was used. Overall, 56 trajectories were run, for 150 steps each (another 150 steps if no reaction was found within the first 150 steps). The electronic structure of the biradical at the PBEh-3c/mSVP level was an unrestricted broken symmetry wave function that showed clear separation of the spin densities between the radicals, positive spin density on one radical and negative spin density on the other one. The five previously selected adsorbed radical geometries were chosen as starting points and for each of these the other radical was placed as relaxed structure in close proximity, $3-5 \AA$ in various starting geometries. Because the interaction between the radicals and the surface is rather weak, we assume that these represent structures accessible by diffusion. In very few cases, non-physical structures were obtained, in which the reactant turned out to be too close to the CO cluster initially. In these cases it flew away rather than reacting. These were not considered in the following. Here, we show that for each binding site both product channels are available, i.e., $\mathrm{CH}_{3} \mathrm{CHO}$ and $\mathrm{CH}_{4}+\mathrm{CO}$ can be formed. Additionally an outcome of the simulation can also be that no reaction takes place.

In principle it is possible to obtain a branching ratio for the two different product channels by sampling sufficient binding sites per binding mode of the first adsorbed radical and running MD simulations for multiple starting geometries with the second radical in close proximity. However, this falls beyond the scope of this work as too many simulations would be necessary to yield a statistically relevant result. 
Table 1: Benchmark of interaction energies (in $\mathbf{K}$ ) of $\mathbf{C O}$ with $\mathbf{C H}_{3}$ and $\mathbf{H C O}$.

\begin{tabular}{l|rr|rr} 
& PBEh-3c & $\begin{array}{r}\text { CCSD(T) } \\
\text { /PBEh-3 }\end{array}$ & M06-2X & $\begin{array}{r}\text { CCSD(T) } \\
\text { /M06-2X }\end{array}$ \\
\hline $\mathrm{HCO}-\mathrm{CO}$ a & 320 & 250 & 245 & 250 \\
$\mathrm{HCO}-\mathrm{CO}$ b & 890 & 485 & 245 & 250 \\
$\mathrm{HCO}-(\mathrm{CO})_{2}$ a & 1100 & 445 & 750 & 550 \\
$\mathrm{HCO}-(\mathrm{CO})_{2}$ b & 1400 & 630 & 820 & 740 \\
$\mathrm{CH}_{3}-\mathrm{CO}$ & 495 & 110 & 665 & 125 \\
$\mathrm{CH}_{3}-(\mathrm{CO})_{2} \mathrm{a}$ & 1355 & 440 & 1045 & 515 \\
$\mathrm{CH}_{3}-(\mathrm{CO})_{2} \mathrm{~b}$ & 1000 & 225 & 1313 & 365
\end{tabular}

The labels a and $\mathrm{b}$ refer to different conformers.

\section{Benchmark of the level of theory}

Given the total system size, between 34 and 41 atoms, we are restricted to using mainly the PBEh-3c/mSVP level of theory. Often the M06-2X functional is seen as a more accurate choice when higher levels of theory, such as $\operatorname{CCSD}(\mathrm{T})-\mathrm{F} 12$, are not available. ${ }^{3435}$ In Table 1 the interaction energies of several smaller models, $\mathrm{CH}_{3}-\mathrm{CO}$ and $\mathrm{HCO}-\mathrm{CO}$ dimers and $\mathrm{CH}_{3}{ }^{-}$ $(\mathrm{CO})_{2}$ and $\mathrm{HCO}-(\mathrm{CO})_{2}$ trimers, are presented to estimate the accuracy for the larger cluster used in the rest of this work. The values for PBEh-3c and M06-2X calculations are obtained following a full optimization routine, whilst the values at the $\operatorname{CCSD}(\mathrm{T})-\mathrm{F} 12$ level of theory refer to energies calculated at the geometries optimized with PBEh-3c and M06-2X. All values are given without zero-point energy correction.

It is immediately clear from the comparison between the DFT and CCSD(T)-F12 values that both functionals tend to overestimate the interaction energy, predict too strong binding. This increases with the number of $\mathrm{CO}$ molecules in the cluster and is largest for the $\mathrm{CH}_{3^{-}}$ $(\mathrm{CO})_{n}$ systems. In other words, the binding energy distributions obtained should be shifted somewhat to lower binding energies. 
Table 2: Ranges of binding energies of $\mathrm{HCO}$ and $\mathrm{CH}_{3}$ to amorphous $\mathrm{CO}$ (this work) compared to water ice.

\begin{tabular}{l|cc|cccc} 
& \multicolumn{2}{|c|}{ amorphous CO } & \multicolumn{2}{c}{ crystalline $\mathrm{H}_{2} \mathrm{O}^{*}$} & single $\mathrm{H}_{2} \mathrm{O}^{\dagger}$ & amorphous $\mathrm{H}_{2} \mathrm{O}^{\ddagger}$ \\
& M06-2X & PBEh-3c & M06-2X & wB97XD & M06 \& MP2 & M06-2X+D3 \\
\hline $\mathrm{HCO}$ & $230-2300$ & $500-2100$ & $1400-4900$ & $350-5000$ & 2400 & 2333 \\
avg. & 1050 & 1250 & & & & 734 \\
$\mathrm{CH}_{3}$ & $175-1900$ & $250-1300$ & $1300-3100$ & $700-3250$ & 1600 & \\
avg. & 1150 & 900 & & & \\
$*$ &
\end{tabular}

\section{Results and Discussion}

\section{$\mathrm{HCO}$ and $\mathrm{CH}_{3}$ binding sites}

Binding energies for 60 binding sites for $\mathrm{HCO}$ and $\mathrm{CH}_{3}$ on the $\mathrm{CO}$ cluster were calculated. The average potential energy for binding was $1050 \mathrm{~K}$ for $\mathrm{HCO}$ and $1150 \mathrm{~K}$ for $\mathrm{CH}_{3}$, both obtained with M06-2X+D3, see Table 2, With PBEh-3c/mSVP the binding is predicted somewhat stronger for $\mathrm{HCO}$ and somewhat weaker for $\mathrm{CH}_{3}$, see also Table 2, Depending on the binding sites, the binding energies are rather broadly distributed, as indicated in Table 2 . This is consistent with findings for other adsorbates on amorphous surfaces. ${ }^{37+39}$ Histograms of the binding energy distributions are provided in the Supporting Information.

These values are compared to the binding energies to water calculated by Sameera et al. 34 for the same radicals on a crystalline water surface, to the results by Wakelam et al. 35 obtained from interaction with a single water molecule, and to the results by Enrique-Romero et al. ${ }^{\sqrt[36]{6}}$ on an amorphous water cluster in Table 2. The interaction of both radicals with a $\mathrm{H}_{2} \mathrm{O}$ surface is clearly stronger than with a CO surface, as expected.

Five representative binding modes were analyzed in more detail. The ZPE was calculated on the PBEh-3c/mSVP level. Subsequently, the geometry was refined at the M062X/TZVPD+D3 level. The resulting binding energies are listed in Table 3. The ZPE correction is on average around $360 \mathrm{~K}$ for both radicals, but varies a bit between the binding sites. The structures are shown in Figure 2, and non-bonded interatomic distances between 


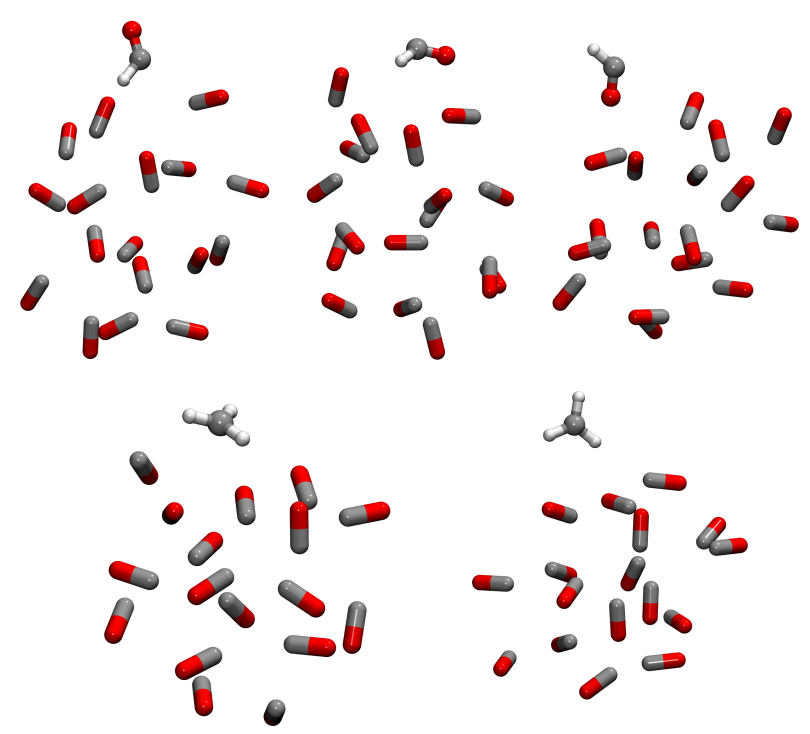

Figure 2: Binding modes of $\mathrm{HCO}$ (top) and $\mathrm{CH}_{3}$ (bottom) to the $\mathrm{CO}$ cluster. The order corresponds to Table 3 .

Table 3: Binding energies (in $\mathrm{K}$ ) of $\mathrm{HCO}$ and $\mathrm{CH}_{3}$ on specific sites of a $\mathrm{CO}$ cluster.

\begin{tabular}{lrrrr} 
& M06-2X+D3 & \multicolumn{3}{c}{ PBEh-3c } \\
& $E_{\text {bind }}$ & $E_{\text {bind }}$ & ZPE & Total \\
\hline $\mathrm{HCO}-\mathrm{H}$ & 915 & 1070 & -350 & 720 \\
$\mathrm{HCO}-$ flat & 1180 & 1410 & -480 & 930 \\
$\mathrm{HCO}-\mathrm{O}$ & $1480^{a}$ & 1250 & -270 & 980 \\
$\mathrm{CH}_{3}$-flat & 1960 & 1140 & -450 & 690 \\
$\mathrm{CH}_{3}$-H & $845^{a}$ & 630 & -270 & 360
\end{tabular}

${ }^{a}$ The reoptimization with M06-2X+D3 lead to a considerable change in the geometry

Table 4: Non-bonded interatomic distances (in $\AA$ ) of typical binding sites of HCO and $\mathrm{CH}_{3}$ on a $\mathrm{CO}$ cluster.

\begin{tabular}{lrrrrr} 
& $\mathrm{H}-\mathrm{O}$ & $\mathrm{H}-\mathrm{O}$ & $\mathrm{H}-\mathrm{O}$ & $\mathrm{O}-\mathrm{C}$ & $\mathrm{O}-\mathrm{C}$ \\
\hline HCO-H & 2.91 & 3.05 & 3.18 & & \\
HCO-flat & 3.03 & 3.04 & 3.07 & & \\
HCO-O & & & & 3.17 & 3.18 \\
\hline & $\mathrm{C}-\mathrm{C}$ & $\mathrm{C}-\mathrm{C}$ & $\mathrm{H}-\mathrm{C}$ & $\mathrm{H}-\mathrm{O}$ & \\
\hline $\mathrm{CH}_{3}$-flat & 3.42 & 3.53 & & & \\
$\mathrm{CH}_{3}-\mathrm{H}$ & & & 2.78 & 3.14 &
\end{tabular}


the adsorbates and the CO cluster are given in Table 4. All of the values are distances between the adsorbate and the nearest $\mathrm{CO}$ molecules of the cluster. The label $\mathrm{O}-\mathrm{C}$ refers to distances from $\mathrm{O}$ of $\mathrm{HCO}$ to $\mathrm{C}$ of the nearest $\mathrm{CO}$ molecules, $\mathrm{C}-\mathrm{C}$ refers to the distance of $\mathrm{C}$ of $\mathrm{CH}_{3}$ to $\mathrm{C}$ of $\mathrm{CO}$. It is clear from the rather large distances, that the adsorbates interact rather weakly with the cluster. The full coordinates of these representative structures are provided in the Supporting Information.

To estimate total binding energies for $\mathrm{HCO}$ and $\mathrm{CH}_{3}$, the average binding energy on the M06-2X+D3 level, the ZPE correction and the shift between M06-2X and CCSD(T)F12 should be taken into account. These result in very rough estimates of average binding energies of $500 \mathrm{~K}$ for $\mathrm{HCO}$ and $200 \mathrm{~K}$ for $\mathrm{CH}_{3}$ on a $\mathrm{CO}$ surface. It should be noted that we find a significant variation between our levels of theory, but even stronger variations between the different binding sites. However, it can be concluded that the interaction of $\mathrm{CO}$ with adsorbed $\mathrm{HCO}$ or $\mathrm{CH}_{3}$ is so weak that diffusion might actually be easy on CO-rich surfaces as our binding energies are in the same range as the diffusion barriers obtained experimentally for $\mathrm{H}$ on $\mathrm{CO} . \underline{40}$

\section{Surface reaction of $\mathrm{HCO}+\mathrm{CH}_{3}$}

For each of the five representative geometries depicted in Figure 2 between 4 and 6 different placements of the second radical were used as initial geometries for MD simulation runs. For each of these sets, we observed both reactive channels, to $\mathrm{CH}_{4}+\mathrm{CO}$ as well as to $\mathrm{CH}_{3} \mathrm{CHO}$. These are indeed barrier-less. The channel observed depends on the orientation of the two radicals with respect to each other. In some cases, no reaction was found. The chosen starting geometries probably lead to small barriers in these cases.

Figure 3 shows the initial geometries (left) and a representative snapshot after the reaction (right) of the MD runs. The CO molecules are shown as sticks, the reactive radicals and their products as balls and sticks. These three trajectories were obtained from the same initial binding mode of $\mathrm{HCO}$ bound flat on the surface. The $\mathrm{CH}_{3}$ moiety was placed at 

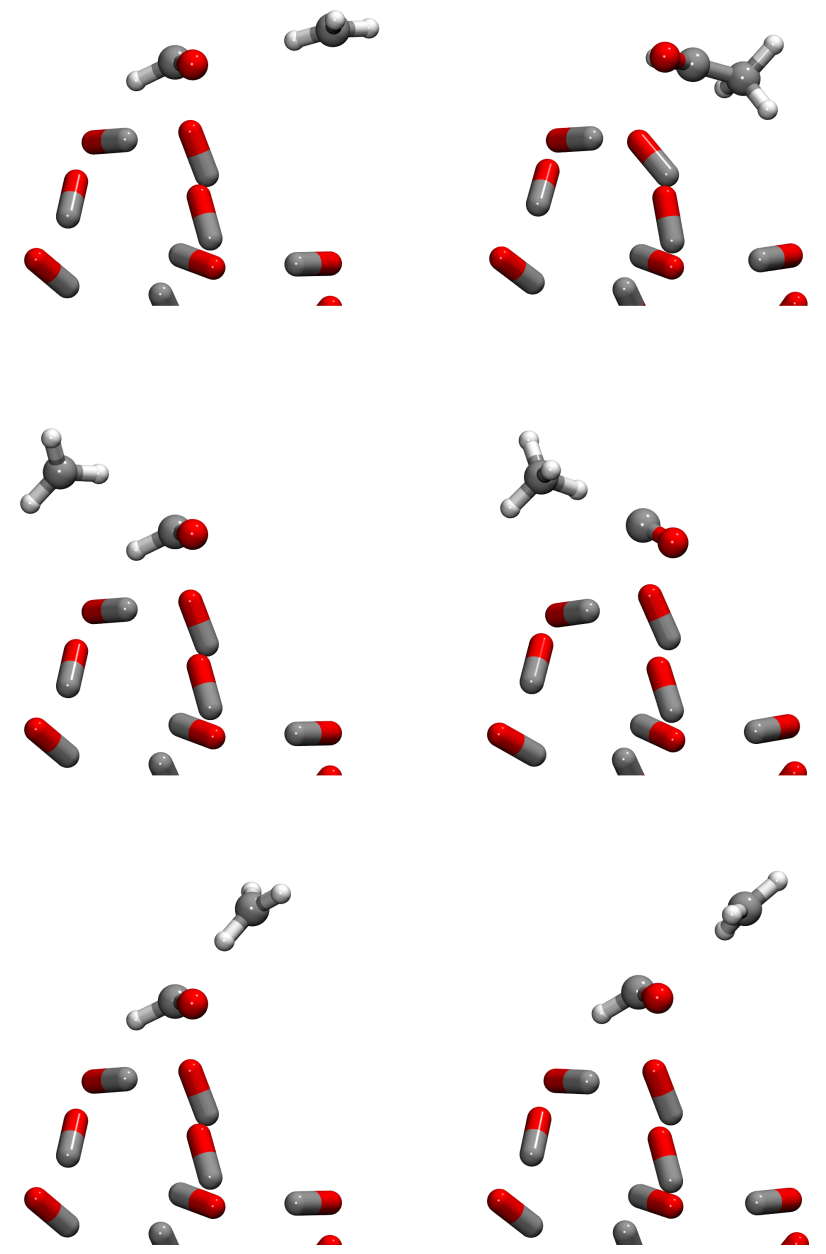

Figure 3: Representative examples of pathways of $\mathrm{HCO}$ and $\mathrm{CH}_{3}$ leading to $\mathrm{CH}_{3} \mathrm{CHO}$ (top), $\mathrm{CH}_{4}+\mathrm{CO}$ (middle) or non-reactive binding of both reactants to the $\mathrm{CO}$ cluster (bottom). 
different positions, leading to different products. Note that given the low binding energies of the radicals with the cluster, the initial geometries, for which the second reactant seems to be somewhat distant from the cluster, are actually realistic. Here we do not consider the reaction of one adsorbed radical with another radical from the gas phase.

For each of the five selected geometries (Figure 2) both $\mathrm{CH}_{4}+\mathrm{CO}$ and $\mathrm{CH}_{3} \mathrm{CHO}$ can be formed. In several cases, the reactants stayed associated with the CO cluster, but did not react.

\section{Conclusions}

We have studied the chemistry of $\mathrm{HCO}$ and $\mathrm{CH}_{3}$ radicals on an amorphous $\mathrm{CO}$ ice surface. The binding energies of both radicals are low, which is understandable given their low dipole moments and the inert character of the CO surface. The binding energies of approximately $500 \mathrm{~K}$ for $\mathrm{HCO}$ and $200 \mathrm{~K}$ for $\mathrm{CH}_{3}$ are comparable to those of hydrogen atoms on the $\mathrm{CO}$ surface. As found for other adsorption processes on amorphous surfaces, a broad distribution of the binding energies of $\mathrm{HCO}$ and $\mathrm{CH}_{3}$ was obtained. The comparison of the levels of theory demonstrated that a thorough benchmarking, the inclusion of vibrational zero point energy, as well as sufficient configurational sampling are necessary for reliable prediction of binding energies. When both radicals are placed close to each other ( 3 to $4 \AA$ ), the relative orientation of the reactants with respect to each other determines which of the products is formed: (a) $\mathrm{CH}_{4}+\mathrm{CO}$, (b) $\mathrm{CH}_{3} \mathrm{CHO}$, or (c) no reaction. Thus, we have clearly shown that the formation of complex organic molecules, like acetaldehyde, is possible from simpler radical species like $\mathrm{HCO}$ and $\mathrm{CH}_{3}$ on a $\mathrm{CO}$ surface. We also found that some reaction products can desorb from the surface. This will, however, depend strongly on the morphology of the ice. On a rougher surface, the molecules are more likely to thermalize and stay bound to the ice. Overall, this study should serve as a proof of principle that radical-radical recombinations can lead to the buildup of complex organic molecules rather than as a quantitative study, 
which would require much more configurational sampling. We hope that this will inspire other theoretical chemists to investigate further similar combinations, which may explain the buildup of larger, more complex organic species in interstellar environments.

\section{Acknowledgments}

Albert Rimola is thanked for fruitful and stimulating discussions. The authors acknowledge support for computer time by the state of Baden-Württemberg through bwHPC and the Germany Research Foundation (DFG) through grant no. INST 40/467-1FUGG. This project was financially supported by the European Unions Horizon 2020 research and innovation programme (grant agreement No. 646717, TUNNELCHEM), the Alexander von Humboldt Foundation, the Netherlands Organisation for Scientific Research (NWO) via a VENI fellowship (722.017.008) and the COST Action CM1401 via an STSM travel grant.

\section{Supporting Information}

Cartesian coordinates of the CO cluster, of representative binding modes, and histograms of the binding energy distributions are provided free of charge on the ACS Publications website.

\section{References}

(1) Gottlieb, C. A. Detection of Acetaldehyde in Sagittarius. Molecules in the galactic environment. 1973; p 181.

(2) Fourikis, N.; Sinclair, M. W.; Robinson, B. J.; Godfrey, P. D.; Brown, R. D. Microwave emission of the $2_{11} \rightarrow 2_{12}$ rotational transition in interstellar acetaldehyde. Aust. J. Phys. 1974, 27, 425. 
(3) Gilmore, W.; Morris, M.; Johnson, D. R.; Lovas, F. J.; Zuckerman, B.; Turner, B. E.; Palmer, P. Observation of the $6_{16}-5_{15}$ transitions of acetaldehyde in Sagittarius B2. Astrophys. J. 1976, 204, 43-46.

(4) Matthews, H. E.; Friberg, P.; Irvine, W. M. The detection of acetaldehyde in cold dust clouds. Astrophys. J. 1985, 290, 609-614.

(5) Turner, B. E.; Terzieva, R.; Herbst, E. The physics and chemistry of small translucent molecular clouds. XII. More complex species explainable by gas-phase processes. Astrophys. J. 1999, 518, 699-732.

(6) Thiel, V.; Belloche, A.; Menten, K. M.; Garrod, R. T.; Müller, H. S. P. Complex organic molecules in diffuse clouds along the line of sight to Sagittarius B2. Astron. Astrophys. 2017, 605, L6.

(7) Belloche, A.; Müller, H. S. P.; Menten, K. M.; Schilke, P.; Comito, C. Complex organic molecules in the interstellar medium: IRAM 30 m line survey of Sagittarius B2(N) and (M). Astron. Astrophys. 2013, 559, A47.

(8) Bacmann, A.; Taquet, V.; Faure, A.; Kahane, C.; Ceccarelli, C. Detection of complex organic molecules in a prestellar core: a new challenge for astrochemical models. Astron. Astrophys. 2012, 541, L12.

(9) Vastel, C.; Ceccarelli, C.; Lefloch, B.; Bachiller, R. The origin of complex organic molecules in prestellar cores. Astrophys. J. Lett. 2014, 795, L2.

(10) Garrod, R. T.; Herbst, E. Formation of methyl formate and other organic species in the warm-up phase of hot molecular cores. Astron. Astrophys. 2006, 457, 927-936.

(11) Woods, P. M.; Slater, B.; Raza, Z.; Viti, S.; Brown, W. A.; Burke, D. J. Glycolaldehyde formation via the dimerization of the formyl radical. Astrophys. J. 2013, 7r7, 90. 
(12) Fedoseev, G.; Cuppen, H. M.; Ioppolo, S.; Lamberts, T.; Linnartz, H. Experimental evidence for glycolaldehyde and ethylene glycol formation by surface hydrogenation of CO molecules under dense molecular cloud conditions. Mon. Notices Royal Astron. Soc. 2015, 448, 1288-1297.

(13) Lamberts, T.; de Vries, X.; Cuppen, H. M. The formation of ice mantles on interstellar grains revisited - the effect of exothermicity. Faraday Disc. 2014, 168, 327.

(14) Rimola, A.; Skouteris, D.; Balucani, N.; Ceccarelli, C.; Enrique-Romero, J.; Taquet, V.; Ugliengo, P. Can formamide be formed on interstellar ice? An atomistic perspective. ACS Earth Space Chem. 2018, 2, 720-734.

(15) Öberg, K. I.; Garrod, R. T.; van Dishoeck, E. F.; Linnartz, H. Formation rates of complex organics in UV irradiated $\mathrm{CH}_{3} \mathrm{OH}$-rich ices. I. Experiments. Astron. Astrophys. 2009, 504, 891-913.

(16) Paardekooper, D. M.; Bossa, J.-B.; Linnartz, H. Laser desorption time-of-flight mass spectrometry of vacuum UV photo-processed methanol ice. Astron. Astrophys. 2016, 592, A67.

(17) Boogert, A. C. A.; Gerakines, P. A.; Whittet, D. C. B. Observations of the icy universe. Annu. Rev. Astron. Astrophys. 2015, 53, 541-581.

(18) Sherwood, P. et al. QUASI: A general purpose implementation of the QM/MM approach and its application to problems in catalysis. J. Mol. Struct. (THEOCHEM) 2003, 632, 1.

(19) Metz, S.; Kästner, J.; Sokol, A. A.; Keal, T. W.; Sherwood, P. ChemShell-a modular software package for QM/MM simulations. WIREs Comput. Mol. Sci. 2014, 4, 101110. 
(20) Kästner, J.; Carr, J. M.; Keal, T. W.; Thiel, W.; Wander, A.; Sherwood, P. DL-FIND: an open-source geometry optimizer for atomistic simulations. J. Phys. Chem. A 2009, 113, 11856-11865.

(21) Grimme, S.; Brandenburg, J. G.; Bannwarth, C.; Hansen, A. Consistent structures and interactions by density functional theory with small atomic orbital basis sets. J. Chem. Phys. 2015, 143, 054107.

(22) TURBOMOLE V7.0 2015, a development of University of Karlsruhe and Forschungszentrum Karlsruhe GmbH, 1989-2007, TURBOMOLE GmbH, since 2007; available from http://www.turbomole.com. accessed Feb 4, 2019.

(23) Zhao, Y.; Truhlar, D. G. The M06 suite of density functionals for main group thermochemistry, thermochemical kinetics, noncovalent interactions, excited states, and transition elements: two new functionals and systematic testing of four M06-class functionals and 12 other functionals. Theor. Chem. Acc. 2008, 120, 215.

(24) Weigend, F.; Häser, M.; Patzelt, H.; Ahlrichs, R. RI-MP2: optimized auxiliary basis sets and demonstration of efficiency. Chem. Phys. Lett. 1998, 294, $143-152$.

(25) Rappoport, D.; Furche, F. Property-optimized Gaussian basis sets for molecular response calculations. J. Chem. Phys. 2010, 133, 134105.

(26) Grimme, S.; Antony, J.; Ehrlich, S.; Krieg, H. A consistent and accurate ab initio parametrization of density functional dispersion correction (DFT-D) for the 94 elements H-Pu. J. Chem. Phys. 2010, 132, 154104.

(27) Knowles, P. J.; Hampel, C.; Werner, H.-J. Coupled cluster theory for high spin, open shell reference wave functions. J. Chem. Phys. 1993, 99, 5219. 
(28) Deegan, M. J. O.; Knowles, P. J. Perturbative corrections to account for triple excitations in closed and open-shell coupled-cluster theories. Chem. Phys. Lett. 1994, 227, $321-326$.

(29) Knizia, G.; Adler, T. B.; Werner, H.-J. Simplified CCSD(T)-F12 methods: Theory and benchmarks. J. Chem. Phys. 2009, 130, 054104.

(30) Adler, T. B.; Knizia, G.; Werner, H.-J. A simple and efficient CCSD(T)-F12 approximation. J. Chem. Phys. 2007, 127, 221106.

(31) Peterson, K. A.; Adler, T. B.; Werner, H.-J. Systematically convergent basis sets for explicitly correlated wavefunctions: The atoms H, He, BNe, and AlAr. J. Chem. Phys. 2008, 128, 084102 .

(32) Werner, H.-J.; Knowles, P. J.; Knizia, G.; Manby, F. R.; Schütz, M. Molpro: a generalpurpose quantum chemistry program package. WIREs Comput. Mol. Sci. 2012, 2, $242-253$.

(33) Hanwell, M. D.; Curtis, D. E.; Lonie, D. C.; Vandermeersch, T.; Zurek, E.; Hutchison, G. R. Avogadro: an advanced semantic chemical editor, visualization, and analysis platform. J. Cheminformatics 2012, 4, 17.

(34) Sameera, W. M. C.; Senevirathne, B.; Andersson, S.; Maseras, F.; Nyman, G. ONIOM(QM:AMOEBA09) Study on binding energies and binding preference of OH, $\mathrm{HCO}$, and $\mathrm{CH}_{3}$ radicals on hexagonal water ice (Ih). J. Phys. Chem. C 2017, 121, $15223-15232$.

(35) Wakelam, V.; Loison, J.-C.; Mereau, R.; Ruaud, M. Binding energies: New values and impact on the efficiency of chemical desorption. Mol. Astrophys. 2017, 6, $22-35$.

(36) Enrique-Romero, J.; Rimola, A.; Ceccarelli, C.; Balucani, N. The (impossible?) forma- 
tion of acetaldehyde on the grain surfaces: insights from quantum chemical calculations. Mon. Notices Royal Astron. Soc. 2016, 459, L6-L10.

(37) Song, L.; Kästner, J. Formation of the prebiotic molecule $\mathrm{NH}_{2} \mathrm{CHO}$ on astronomical amorphous solid water surfaces: accurate tunneling rate calculations. Phys. Chem. Chem. Phys. 2016, 18, 29278-29285.

(38) Lamberts, T.; Kästner, J. Influence of surface and bulk water ice on the reactivity of a water-forming reaction. Astrophys. J. 2017, 846, 43.

(39) Song, L.; Kästner, J. Tunneling rate constants for $\mathrm{H}_{2} \mathrm{CO}+\mathrm{H}$ on amorphous solid water surfaces. Astrophys. J. 2017, 850, 118.

(40) Kimura, Y.; Tsuge, M.; Pirronello, V.; Kouchi, A.; Watanabe, N. Measurements of the activation energies for atomic hydrogen diffusion on pure solid CO. Astrophys. J. Lett. 2018, 858, L23. 
Table of Contents only:

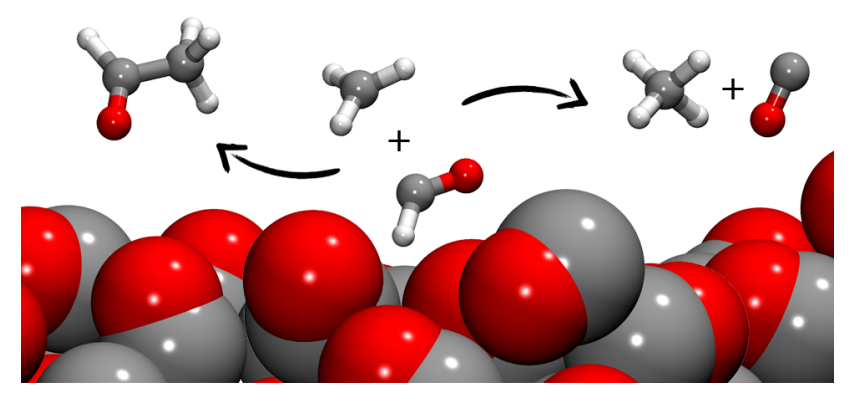

